\title{
Stellar molecular jets traced by maser emission
}

\author{
Hiroshi Imai \\ Department of Physics, Faculty of Science, Kagoshima University, Japan \\ email: hiroimai@sci.kagoshima-u.ac.jp
}

\begin{abstract}
Highly collimated jets found in AGB and post-AGB stars are expected to play an important role for shaping planetary nebulae. Recent VLBI observations of $\mathrm{H}_{2} \mathrm{O}$ maser sources have revealed that some of the spatio-kinematical structures of $\mathrm{H}_{2} \mathrm{O}$ maser sources exhibit stellar jets with extremely spatially and kinematically high collimation. Such stellar $\mathrm{H}_{2} \mathrm{O}$ maser jets, so-called "water fountains", have been identified in about 10 sources to date. Here we review recent VLBI observations and relevant observational results of the water fountain sources. They have revealed a typical dynamical age and the detailed kinematical structures of the water fountains, the possibility of the existence of "equatorial flows", and the evolutionary status of the host stars. The location and kinematics of one of the water fountain sources in the Galaxy is also revealed and shown here.
\end{abstract}

Keywords. masers, stars:AGB and post-AGB, mass loss, winds, outflows

\section{Introduction}

A stellar jet appearing at the final stage of stellar evolution is a manifestation of the important phenomena that form asymmetric planetary nebulae $(\mathrm{PNe})$. Based on the large incidence of bipolar and multipolar optical morphologies and the common presence of point-symmetry found in a sample of young PNe, Sahai \& Trauger (1998) concluded that collimated jets have been operational in a large fraction of PNe, and expected to be one of the major factors shaping these objects. Similar morphologies have been found in a survey of pre-PNe (Sahai 2004), leading to the current paradigm that such a collimated jet had already been launched at an earlier phase of stellar evolution - the asymptotic giant branch (AGB) phase - and is frequently observable in molecular line emission. Subsequently an optically visible PN is seen at the post-AGB phase.

There are candidate sources that show us the earliest stage of stellar jet emergence. "Water fountains" are the most promising candidates; they exhibit extremely-highvelocity flows traced by $\mathrm{H}_{2} \mathrm{O}$ maser emission. The outflow velocity is much higher than the typical expansion velocity of circumstellar envelopes of AGB stars found in $1612 \mathrm{MHz}$ $\mathrm{OH}$ masers (10-25 $\mathrm{km} \mathrm{s}^{-1}$, e.g. te Lintel Hekkert et al. 1989). The $\mathrm{H}_{2} \mathrm{O}$ maser emission exhibits high collimation in both its morphology and kinematics. This suggests that high density gas is supplied from the vicinity of the stellar surface to the jet tips where $\mathrm{H}_{2} \mathrm{O}$ maser emission is excited (e.g., Elitzur et al. 1989). Because VLBI observations have been made for some of these water fountains, it is possible to undertake statistical analysis to estimate typical values of their physical parameters, which leads to understanding the final evolution of the circumstellar envelopes and the formation mechanism of stellar jets.

Note that high precision astrometry of $\mathrm{H}_{2} \mathrm{O}$ masers in the water fountains provides great opportunity to directly estimate the locations and kinematics of the water fountains 
Table 1. Parameters of "water fountain" sources.

\begin{tabular}{|c|c|c|c|c|c|c|}
\hline IRAS name & Other name & $\begin{array}{c}\text { Length } \\
(\operatorname{arcsec})\end{array}$ & $\begin{array}{c}\Delta V_{\operatorname{los}} \dagger \\
\left(\mathrm{km} \mathrm{s}^{-1}\right)\end{array}$ & $\begin{array}{c}t_{\text {jet } \ddagger} \\
\text { (year) }\end{array}$ & $\begin{array}{c}\text { Distance } \\
\quad(\mathrm{kpc})\end{array}$ & Reference \\
\hline $15445-5449$ & OH $326.5-0.4$ & & 91 & & & 4 \\
\hline $15544-5332$ & OH $325.8-0.3$ & & 40 & & & 4 \\
\hline $16342-3814$ & $\mathrm{OH} 344.1+5.8$ & 2.4 & 240 & $\sim 100$ & 2 & $3,15,16,17,18$ \\
\hline $16552-3050$ & GLMP 498 & & 170 & & & 19 \\
\hline $18043-2116$ & ОН $0.9-0.4$ & & 204 & & & 4 \\
\hline $18139-1816$ & OH $12.8-0.9$ & 0.11 & 55 & 70 & $?$ & $1,2,10$ \\
\hline $18286-0959$ & & 0.24 & 200 & $\sim 15$ & 3.1 & 5,11 \\
\hline $18450-0148$ & $\mathrm{~W} 43 \mathrm{~A}, \mathrm{OH} 31.0+0.0$ & 0.8 & 190 & 50 & 2.6 & $6,8,13,15,16,20$ \\
\hline $18460-0151$ & $\mathrm{OH} 31.0-0.2$ & 0.11 & 290 & $\sim 5$ & 6.8 & 5,11 \\
\hline $18596+0315$ & OH $37.1-0.8$ & & 59 & & & 4 \\
\hline $19134+2131$ & & 0.15 & 100 & 40 & 8.0 & $12,14,16$ \\
\hline
\end{tabular}

$\dagger$ Full range of the line-of-sight velocities of maser emission.

$\ddagger$ Dynamical age of the jet.

91: Baud et al. 1979, 2: Boboltz \& Marvel 2005, 3: Claussen et al. 2004 4: Deacon et al. 2007, 5: Deguchi et al. 2007, 6: Diamond et al. 1985, 7: Engels 2002, 8: Genzel \& Downes 1977, 9: Engels et al. 1986, 10: Gómez et al. 1994, 11: Imai et al. 2007 in preparation, 12: Imai, Sahai, \& Morris 2007, 13: Imai et al. 2005, 14: Imai et al. 2004, 15] Imai et al. 2002, 16: Likkel et al. 1992, 17: Morris et al. 2003, 18: Sahai et al. 1999, 19: Suárez, Gómez, \& Morata 2007, 20: Vlemmings et al. 2006

in the Galaxy, leading to estimates of masses and the birth places of the stars driving these water fountains.

\section{Lifetime and timing of the water fountain}

Table 1 gives physical parameters of the water fountain jets derived from single-dish and interferometric observations. Lengths of stellar jets traced by $\mathrm{H}_{2} \mathrm{O}$ maser emission range over $6000 \mathrm{AU}$ (in IRAS 16342-3814, Morris et al. 2003, Claussen et al. 2004), which is still equal to or shorter than the radius of a circumstellar envelope of an AGB star (e.g., Elitzur 1992). On the other hand, the flow velocities $V_{\text {flow }} \sim \Delta V_{\text {los }} / 2$ are sometimes over $100 \mathrm{~km} \mathrm{~s}^{-1}$. These give dynamical ages of jets shorter than 100 years, only $\sim 5$ years in an extreme case (Imai et al., in preparation). In the time interval of 100 years, a stellar jet traveling with a typical water fountain velocity of $100 \mathrm{~km} \mathrm{~s}^{-1}$ reaches $\sim 2000 \mathrm{AU}$ from the central star, which corresponds to the typical size of an $\mathrm{OH}$ maser shell in a circumstellar envelope around an $\mathrm{OH} / \mathrm{IR}$ star, at the final stage of energetic mass loss. The $\mathrm{H}_{2} \mathrm{O}$ maser emission is excited near the tip of the stellar jet where high compression takes place in the shock at the interface between the jet and the ambient circumstellar gas. But because the ambient gas density declines with radius, the maser emission may be quenched at the distance where the post-shock gas density falls below a critical value of $n_{\mathrm{H}_{2}} \sim 10^{6} \mathrm{~cm}^{-3}$ (Elitzur et al. 1989).

Note that while optical lobes are observed in some water fountains (IRAS 16342-3814, and IRAS 19134+2131), the optical emission is not detected in others (W43A and OH12.8-0.9). This suggests that the presence of water fountains may help in the identification of new pre-PNe, that may be optically invisible due to heavy circumstellar extinction. The optical lobes in IRAS $19134+2131$ and IRAS $16342-3814$ coexist with the $\mathrm{H}_{2} \mathrm{O}$ maser emission (Sahai et al. 1999, Morris et al. 2003, Imai, Sahai, \& Morris 2007). It is expected that the lobes have been recently formed along the collimated fast jet, in which the circumstellar envelope around the evolved star has been penetrated by 
the jet and starlight. These sources may be at a similar stage in the transition from an AGB to post-AGB star.

Does the size of a circumstellar envelope determine the lifetime of a water fountain? We already know that $\mathrm{H}_{2} \mathrm{O}$ masers can survive until the photoionization of the circumstellar envelope starts to form a PN, such as K3-35 (Miranda et al. 2001). In K3-35, $\mathrm{H}_{2} \mathrm{O}$ maser emission is seen at the tips of the radio lobes as well as in the inner and denser part of the circumstellar envelope where the UV radiation from the star has not yet penetrated. However, the maser emission at the lobe tips of K3-35 is only weakly collimated, both morphologically and kinematically, implying that an initially collimated jet traceable by $\mathrm{H}_{2} \mathrm{O}$ maser emission is being destroyed. The dynamical age of the radio lobes is estimated to be 800 yr (Miranda et al. 2001), which may correspond to an upper limit to the lifetime of a water fountain source but it is one order of magnitude higher than the dynamical age estimated using VLBI observations of the maser spatio-kinematical structures.

Here we speculate that the collimated jet may drill through the circumstellar envelope formed during the AGB phase and the tip of the jet may reach the outer surface of the envelope before the onset of photoionization of the envelope. The masers lying beyond the optical lobes, such as those in IRAS 16342-3814, might soon be quenched within a few decades or centuries owing to the decline of the gas density in the pre-shock gas as the jet propagates outward. This in turn leads to a loss of collimation and ultimately to a loss of the maser inversion as the post-shock gas density declines.

\section{Equatorial flows in the water fountain sources}

Highly collimated fast stellar jets as described above are expected to be driven by magneto-hydrodynamical processes (e.g., Blackman et al. 2001, Vlemmings et al. 2006). However it is unclear whether the stellar object driving the jet is a single star or a binary system; one is an AGB star providing material to another through an accretion disk. Astrometry for the $\mathrm{H}_{2} \mathrm{O}$ and $1612 \mathrm{MHz} \mathrm{OH}$ maser emission will provide an important clue to find each of the locations of the driving sources of the collimated jet and the AGB flow. Only W43A has SiO maser emission (Nakashima \& Deguchi 2003, Imai et al. 2005), which provides a better opportunity of exactly finding the location of the AGB star.

Figure 1 compares the locations of the dynamical centers of the $\mathrm{H}_{2} \mathrm{O}$ maser jet and the $\mathrm{SiO}$ maser flow using a common extragalactic position reference. Although astrometric accuracy is poorer in the north-south direction because W43A is located very close to the celestial equator, the dynamical centers coincide within 4 mas (10 AU at $2.6 \mathrm{kpc}$ ). The $\mathrm{SiO}$ maser flow is well modeled by a biconically expanding flow with a low velocity $\left(\sim 20 \mathrm{~km} \mathrm{~s}^{-1}\right)$ and a large opening angle (Imai et al. 2005$)$. These implies that both of the collimated jet and the biconical flow are driven in the region within $10 \mathrm{AU}$.

Note that the spatio-kinematical structure of the $\mathrm{SiO}$ masers does not exhibit any rotation or contraction as suggested when mass accretion occurs from a disk. Figure 2 shows relative proper motions of the W43A $\mathrm{H}_{2} \mathrm{O}$ masers. We found a maser feature that is located close to the dynamical center and has a Doppler velocity close to the systemic velocity of W43A $\left(V_{\mathrm{LSR}}=34 \mathrm{~km} \mathrm{~s}^{-1}\right)$ and a proper motion corresponding to a speed of $\sim 30 \mathrm{~km} \mathrm{~s}^{-1}$. The velocity vector is pointed to the different direction from the jet major axis. The low velocity maser feature is expected to be associated with an "equatorial" flow, rather than an accretion disk. Such equatorial flows have recently been recognized in other water fountain sources, IRAS 18286-0959 and IRAS 18460-0151, as well (Deguchi et al. 2007). Figure 3 and 4 shows the spatio-kinematics of IRAS 18460-0151 and IRAS 18286-0959, respectively. The former source has the fastest jet ever observed with a speed over $300 \mathrm{~km} \mathrm{~s}^{-1}$. This source also has an equatorial flow with a velocity of $\sim 30 \mathrm{~km} \mathrm{~s}^{-1}$ in the region close to the midpoint of the most blue-shifted and red-shifted 


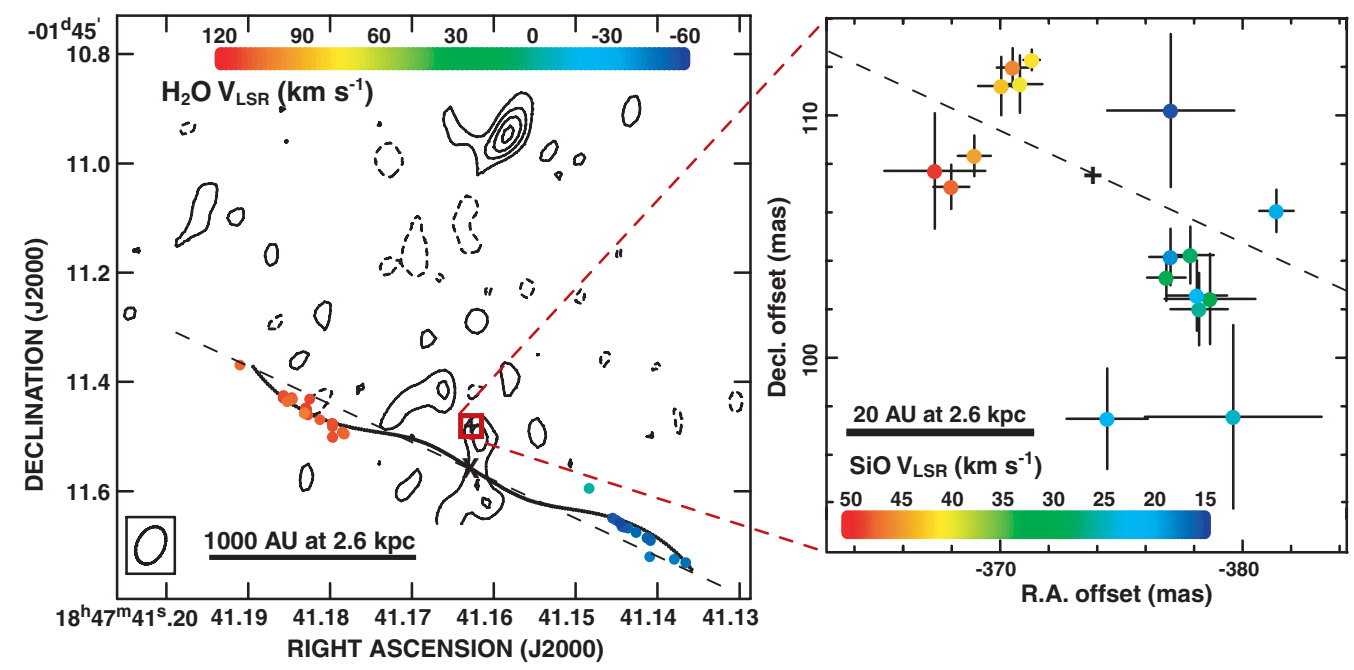

Figure 1. $\mathrm{H}_{2} \mathrm{O}, \mathrm{SiO}(v=1, J=1-0)$ maser emission, and $7 \mathrm{~mm}$ continuum emission in W43A. Different colors denote the maser feature line-of-sight velocities, according to the color scale. Left Spatio-kinematics of the $\mathrm{H}_{2} \mathrm{O}$ maser features observed on 2002 April 3. A dotted line shows a pattern of a precessing jet appearing in the year 2002.3, which is modeled by Imai et al. 2002. A symbol "X" indicates the dynamical center of the modeled jet. A thin dashed line indicates the axis direction of the $\mathrm{H}_{2} \mathrm{O}$ maser jet. Right Spatio-kinematics of the $\mathrm{SiO}$ maser features. Horizontal and vertical bars of individual maser features indicate uncertainties of feature positions in R.A. and declination, respectively. A plus symbol indicates a roughly-estimated location of the dynamical center of the biconically-expanding flow found in the $\mathrm{SiO}$ maser kinematics. Roughly a flux density of a spot is inversely proportional to the error bar of the spot.
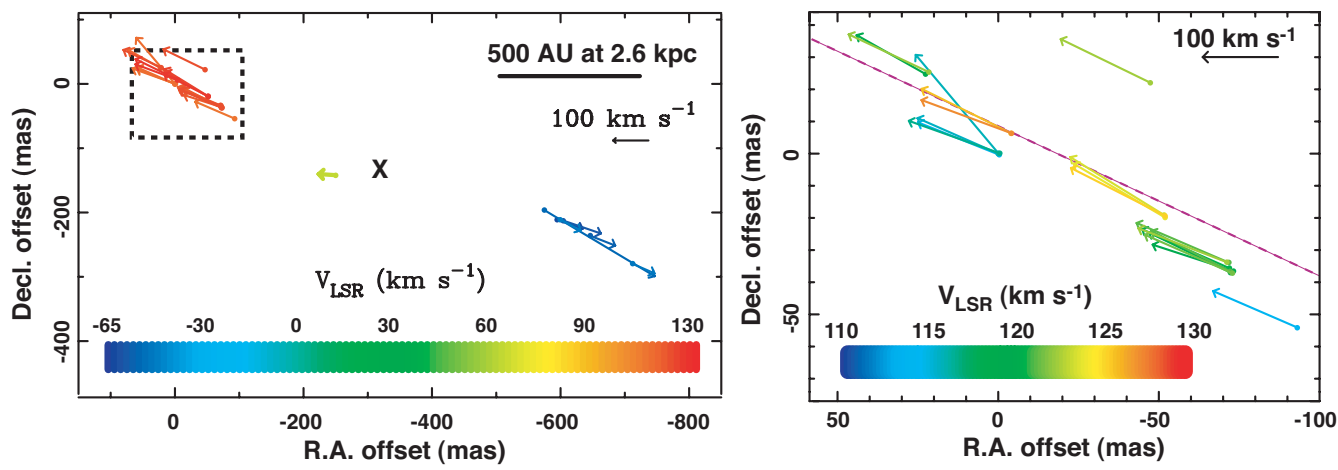

Figure 2. Relative proper motions of $\mathrm{H}_{2} \mathrm{O}$ masers in W43A measured during 2003-2004 with a mean proper motion subtracted. A cross indicates the location of the dynamical center of the precessing bipolar jet. Left the whole distribution of maser features. Right zoom-up view of the feature distribution in the area displayed in a dashed-line box in the left panel.

maser features forming the high velocity jet. The latter source has many velocity maser components, only the most blue-shifted and red-shifted maser features are clearly separated from each other and each of them is distributed in a small area. These imply the coexistence of a collimated fast flow and a slow outflow associated with either an AGB envelope or an equatorial flow driven by the fast flow as its sub-product. The existence of such equatorial flows may support a hypothesis that a single star can create both the fast collimated and the slow equatorial flows when the star is collapsing into a white dwarf (H. Washimi and S. Miyaji, in private communication). 

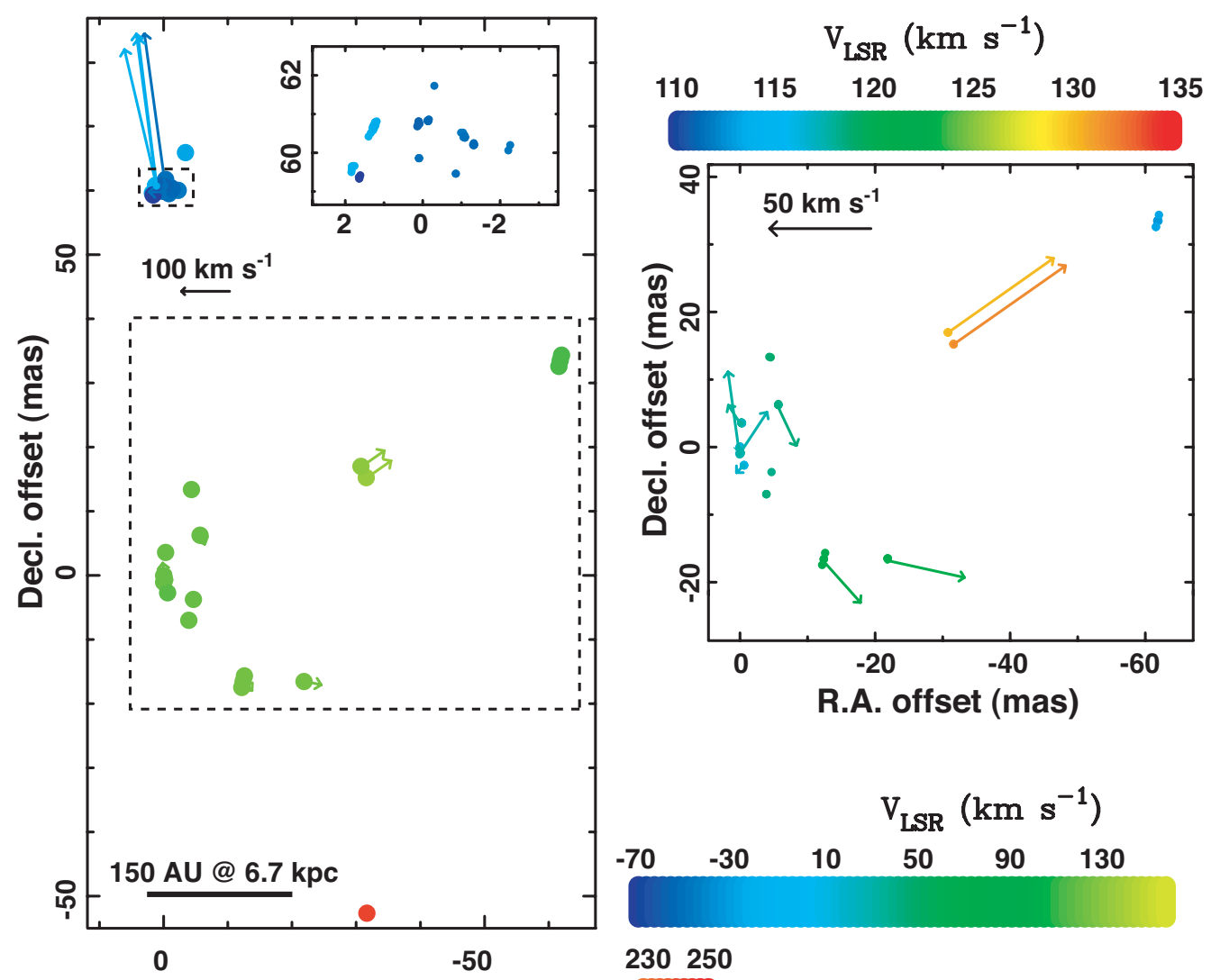

R.A. offset (mas)

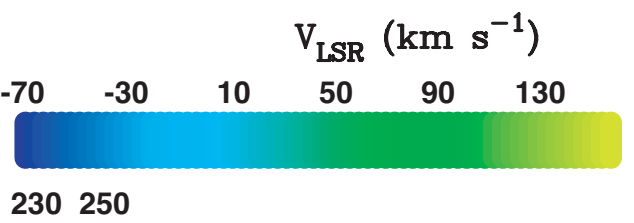

Figure 3. Distribution (filled circle) and relative proper motions (arrows) of $\mathrm{H}_{2} \mathrm{O}$ masers in IRAS 18460-0151 during 2006 September-November. Left Overview of the distribution. Right Zoom-up view of the area displayed with a dashed line box in the left panel. Different colors denote the features' line-of-sight velocities, according to the color scale above this panel. Relative proper motions of maser features are measured with respect to the maser feature located at the origin and biased in the north-west direction by $\sim 30 \mathrm{~km} \mathrm{~s}^{-1}$. Color renditions of the figures can be found in the online version of this paper.

\section{Ballistic corkscrew jets}

The morphology of water fountains may provide a unique clue of the ejection of the material in the jets, leading to a characterization of the central stars. Some of the water fountain jets exhibit spiral patterns. In particular, such a pattern is clearly seen in the distributions of $\mathrm{H}_{2} \mathrm{O}$ masers in W43A (Imai et al. 2002, Imai et al. 2005). On the other hand, a "corkscrew jet" is found in optical emission in IRAS 16342-3814 (Sahai et al. 2005). Recently we found twin spiral patterns of the $\mathrm{H}_{2} \mathrm{O}$ maser distribution in W43A (see figure 1), one of the spiral is seen in the axisymetrically opposite side of another (dotted line) with respect to the jet major axis (thin dashed line). However, curved motions of the maser features are not recognized. The right panels of figure 2 and figure 5 show details of the proper motions of the $\mathrm{H}_{2} \mathrm{O}$ masers found in W43A and IRAS $19134+2131$, respectively. In each source, we find an alignment of maser features whose direction has an offset from the major axis of the jet. On the other hand, proper motion vectors of maser features are still parallel to the jet axis. These suggest that the corkscrew jets found in these sources are formed by ballistic motions of maser features from the driving source of 


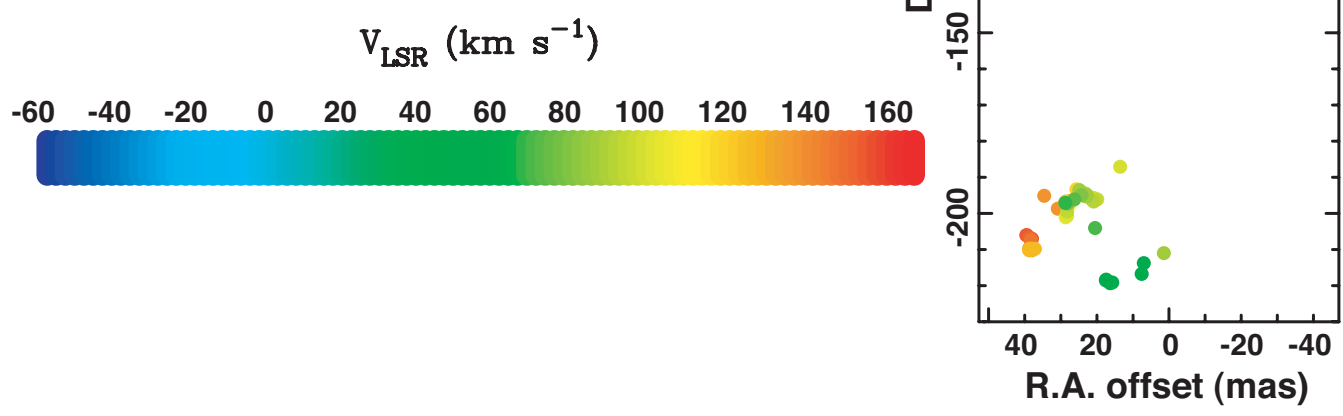

Figure 4. Same as figure 3, but for IRAS 18286-0959 observed on 2006 September 14.

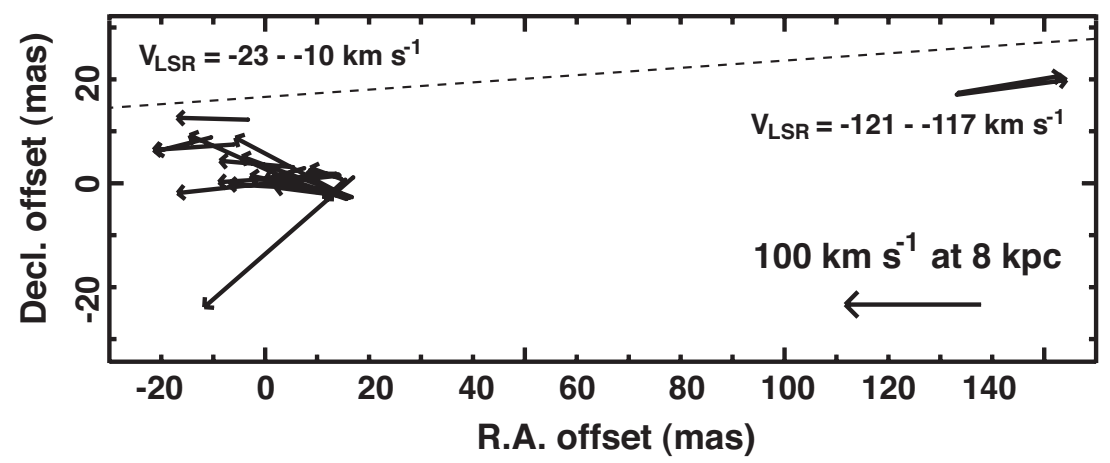

Figure 5. $\mathrm{H}_{2} \mathrm{O}$ maser feature distribution in IRAS $19134+2131$ (roots of arrows) and relative proper motion vectors of these masers (arrows) with respect to a position reference maser feature with a mean proper motion subtracted.

the jets, but whose directions are precessing with time. The alignments of maser features may be formed at the shock fronts interfaced with the ambient circumstellar envelopes.

\section{Location and motion of the water fountain in the Galaxy}

The absolute coordinates of $\mathrm{H}_{2} \mathrm{O}$ masers associated with IRAS 19134+2131 (hereafter I19134) have been measured with respect to the extragalactic position reference source J1925+2106 for a span over one year (Imai, Sahai, \& Morris 2007). We therefore fit the temporal variation of the mean position of three maser features to a kinematical model 

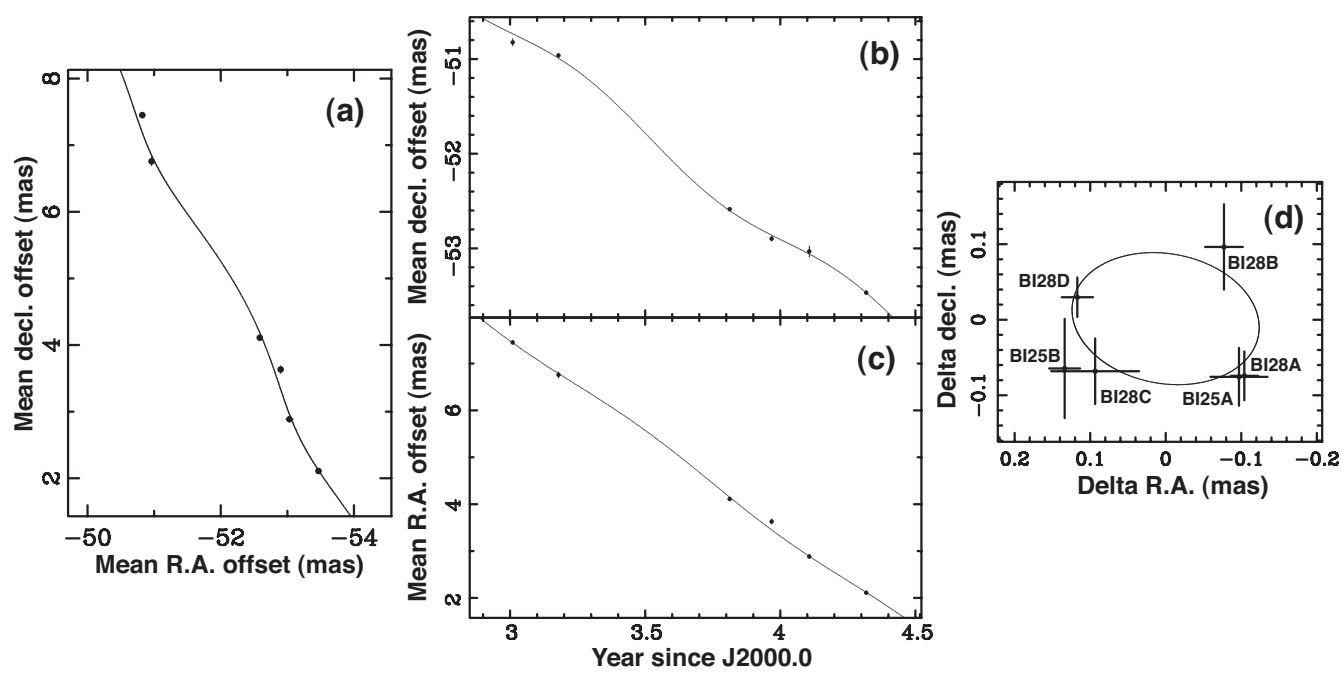

Figure 6. Mean motion of three $\mathrm{H}_{2} \mathrm{O}$ maser features in IRAS $19134+2131$ with respect to the extragalactic position reference source J1925+2106. (a) Mean R.A. and decl. offsets on the sky with respect to the phase-tracking center. $(b)$ and $(c)$ Mean R.A. and decl. offsets against time. (d) Relative mean offsets with a mean proper motion subtracted. The observation code is shown alongside each measured position.

consisting of an annual parallax and a constant velocity mean secular motion. Figure 6 shows the fitting result. We obtain a best-fit annual parallax, corresponding to a distance to $\mathrm{I} 19134$ of $D=8.0_{-0.7}^{+0.9} \mathrm{kpc}$. On the basis of the inferred heliocentric distance and the systemic secular maser motion, we estimate the location of I19134 in the Galaxy to be $[R, \theta, z]=\left[7.4_{-0.3}^{+0.4} \mathrm{kpc}, 62^{\circ} \pm 5^{\circ}, 0.65_{-0.06}^{+0.07} \mathrm{kpc}\right]$, and the $3-\mathrm{D}$ velocity vector in Galactic cylindrical coordinates to be, $\left[V_{R}, V_{\theta}, V_{z}\right]=\left[3_{-46}^{+53}, 125_{-28}^{+20}, 8_{-39}^{+48}\right)\left[\mathrm{km} \mathrm{s}^{-1}\right]$.

The estimated location is close to the Perseus spiral arm within $\sim 1 \mathrm{kpc}$ (c.f., Nakanishi $\&$ Sofue 2006). Note that the estimated Galactic rotation velocity of I19134 $\left(\sim 125 \mathrm{~km} \mathrm{~s}^{-1}\right)$ is much slower than that implied by the Galactic rotation curve $\left(\sim 220 \mathrm{~km} \mathrm{~s}^{-1}\right.$, e.g. Clemens 1985, Dehnen \& Binney 1998). It implies that the I19134 orbit must have large excursions in radius although I19134 is orbiting along a circle at present. I19134 is apparently orbiting as a member of the Galactic "thick" component rather than the "thin" component. Adopting a source velocity perpendicular to the Galactic plane $V_{z}=8 \mathrm{~km} \mathrm{~s}^{-1}$, we find that the present Galactic altitude gives a lower limit to the travel time of I19134 from the Galactic plane to the present position to be $\sim 2.4 \times 10^{7} \mathrm{yr}$. This suggests that the stellar object of I19134 has a lifetime of at least this travel time and a stellar mass smaller than $\sim 8.6 M_{\odot}$ (here we adopt a stellar lifetime to be $t_{*} \propto M_{*} / L_{*} \propto M_{*}^{-2.8}$ ) if the central star of I19134 was born in the Galactic plane as a member of the Galactic "thin disk" component. The statistical study of Galactic PNe by Manchado 2004 shows that the Galactic scale height of bipolar PNe such as I19134 is $\langle z\rangle=100$ pc, suggesting that their progenitors should be high mass stars. Although the mass of I19134's progenitor is therefore expected to be near the upper mass limit estimated above, the present galactic altitude of I19134 and its anomalously slow galactic rotation velocity are quite odd. These may imply an abnormal environment during the birth of I19134's central star. However, the estimated mass of I19134 still has a big uncertainty due to the poor accuracy of the vertical velocity, $V_{z}$. 
Acknowledgements The present work have been done in collaborations with S. Deguchi, P. J. Diamond, S. Kwok, A. Miyazaki, M. Morris, J. Nakashima, K. Obara, T. Omodaka, R. Sahai, T. Sasao, and W. H. T. Vlemmings. The NRAO VLBA and VLA are facilities of the National Science Foundation are operated under a cooperative agreement with Associated Universities, Inc. The EVN is a joint facility of European, Chinese, South African and other radio astronomy institutes funded by their national research councils. H.I. was supported by an IAU Grant for traveling to the symposium and a Grant-in-Aid for Scientific Research from the Japan Society for the Promotion of Science (18740109).

\section{References}

Baud, B., Habing, H. J., Mattheus, H. E., \& Winnberg, A. 1979, A\& $A S$, 36, 193

Blackman, E. G., Frank, A., Markiel, J. A., Thomas, J. H., \& Van Horn, H. M. 2001, Nature, 409,485

Boboltz, D. A., \& Marvel, K. B. 2005, ApJ, 627, L45

Claussen, M., Sahai, R., \& Morris, M. 2004, in ASP Conf. Ser. 313, Asymmetrical Planetary Nebulae III: Winds, Structure and the Thunderbird, ed. M. Meixner et al. (San Francisco: ASP), 331

Clemens, D. P. 1985, ApJ, 295, 422

Deacon, R. M., Chapman, J. M., Green, A. J., \& Sevenster, M. N. 2007, ApJ, 658, 1096

Deguchi, S., Nakashima, J, \& Kwok, S. 2007, ApJ, in preparation

Dehnen, W., \& Binney, J. 1998, MNRAS, 294, 429

Diamond, P. J., Norris, R. P., Rowland, P. R., Booth, R. S., \& Nyman, L.-A. 1985, MNRAS, 211,1

Engels, D. 2002, $A \& A, 388,252$

Engels, D., Schmid-Burgk, J., \& Walmsley, C. M. 1986, A\& A, 67, 129

Elitzur, M. 1992, in Astronomical Masers (Dordrecht: Kluwer)

Elitzur, M., Hollenbach, D. J., \& McKee, C. F. 1989, ApJ, 346, 983

Genzel, R., \& Downes, D. 1977, A\& As, 30, 145

Gómez, Y., Rodríguez, L. F., Contréras, M. E., \& Moran, J. M. 1994, Rev. Mex. AA, 28, 97

Imai, H., Sahai, R., \& Morris, M. 2007, ApJ, submitted

Imai, H., Nakashima, J., Diamond, P. J., Miyazaki, A., \& Deguchi, S. 2005 ApJ, 622, L125

Imai, H., Morris, M., Sahai, R., Hachisuka, K., \& Azzollini, J. R. F. 2004, A\&3A, 420, 265

Imai, H., Obara, K., Diamond, P. J., Omodaka, T., \& Sasao, T. 2002, Nature, 417, 829

Likkel, L., Morris, M., \& Maddalena, R. J. 1992, A\& A, 256, 581

te Lintel Hekkert, P., Versteege-Hensel, H. A., Habing, H. J., \& Wiertz, M. 1989, A\&GAS, 78, 399

Manchado, A. 2004, in ASP Conf. Ser. 313, Asymmetrical Planetary Nebulae III: Winds, Structure and the Thunderbird, ed. M. Meixner et al. (San Francisco: ASP), 3

Miranda, L. F., Gómez, Y., Anglada, G., \& Torrelles, J. M. 2001, Nature, 414, 284

Morris, M. R., Sahai, R., \& Claussen, M. 2003, Rev. Mex. AA Conf., 15, 20

Nakanishi, H., \& Sofue, Y. 2006, PASJ, 58, 847

Nakashima, J., \& Deguchi, S. 2003, PASJ, 55, 229

Sahai, R., Le Mignant, D., Sánchez Contreras, C., Campbell, R. D., \& Chaffee, F. H. 2005, ApJL, 622, L53

Sahai, R. 2004, in ASP Conf. Ser. 313, Asymmetrical Planetary Nebulae III: Winds, Structure and the Thunderbird, ed. M. Meixner et al. (San Francisco: ASP), 141

Sahai, R., \& Nyman, L.-Å. 2000, ApJ, 537, L145

Sahai, R., te Lintel Hellert, P., Morris, M., Zijlstra, A., \& Likkel, L. 1999, ApJ, 514, L115

Sahai, R., \& Trauger, J. 1998, AJ, 116, 1357

Suárez, O., Gómez, J. F., \& Morata, O. 2007, A\&\&A, in press (astro-ph/0703502)

Vlemmings, W. H. T., Diamond, P. J., \& Imai, H. 2006, Nature, 440, 58 